\title{
Computed Results for the Reduction of Iron Oxide Pellets in Moving Beds with Non-uniform Gas and Solids Flow*
}

\author{
By Jun-ichiro YAGI** and Julian SZEKEL $Y^{* * * *}$
}

\section{Synopsis}

A comprehensive set of computed results is presented, describing the reduction of iron oxides with hydrogen in a counterflow packed bed arrangement, when both the gas and the solid streams may be maldistributed for both isothermal and non-isothermal conditions. This maldistribution is generated by assigning a radial dependence to both the void fraction and the particle diameter.

It was possible to examine cases where preferential flow of the gas occurred in the wall region, while preferential mass flow of the solids took place in the central core and vice versa.

It was found that flow maldistribution may have a dramatic effect on the performance of the packed bed reactor by causing a marked distortion of the concentration isopleths. When the solid and the gaseous streams are mismatched, local reastant starvation may occur, which does not allow the reactions to attain completion, notwithstanding the fact that the overall stoichiometric requirements are met by the system.

\section{Introduction}

In the previous paper ${ }^{11}$ a general formulation was presented, describing the reduction of iron oxide pellets, contacted with a hydrogen stream in a countercurrent moving bed arrangement. The formulation was general, in the statement of the problem allowance was made for the non-uniform flow of both the gaseous and the solid streams and for nonisothermal behavior.

Our purpose in the present paper is to present a comprehensive set of the computed results, for the following three specific cases:

1) Spatially uniform gas flow and isothermal conditions, which represents the standard case to which the others will be compared

2) Non-uniform gas flow, but isothermal condition

3) Non-isothermal condition and distributed gas flow

\section{Computed Results}

Let us consider two groups of cases, within the subdivisions detailed above, by assigning a particular radial distribution to the void fraction and to the diameter of the solid particles:

Case (A)

$$
\varepsilon_{v}=0.45-0.1 r \quad 0 \leq r \leq 1
$$

and

$$
d_{p}=0.016-0.006 r \quad 0 \leq r \leq 1
$$

Case (B)

$$
\varepsilon_{v}=0.35+0.1 r \quad 0 \leq r \leq 1
$$

and

$$
d_{p}=0.01+0.006 r \quad 0 \leq r \leq 1
$$

Where $\varepsilon_{v}$ and $d_{p}$ are the void fraction of the bed and the particle diameter respectively and $r$ is the dimensionless radial coordinate, measured from the centerline of the bed.

For case (A), both the void fraction and the particle size decrease from the center toward the wall, thus from fluid flow considerations one would expect preferential flow in the central portion of the bed. The reverse would be true for case (B).

We note that for case (2) the fluid flow field is affected by both the distributed resistance of the bed and by the progress of the chemical reaction, which modifies the gas composition and hence causes changes in the local mass velocity of the gas. In case (3) the flow field of the gas stream is affected by these variables, but also by the changes in the gas temperature. As will be seen subsequently, the interplay of these factors may give rise to some very interesting effects.

The mass velocity (mass flux) of the solids at the entry is given by:

$$
G_{s 0}^{\prime}=u_{s} \rho_{a}\left(1-\varepsilon_{v}\right)
$$

Since $\varepsilon_{v}=f(r), G_{s 0}^{\prime}$ is radially distributed at the inlet, even if $u_{s}$ is assumed to be uniform. ${ }^{\dagger}$ As the solid charge proceeds through the bed $G_{s z}$ will be a function of both the axial and the radial coordinates, because the variation in the pellet density will be brought about by the chemical reaction. We note here that in the presentation of the results $X_{1}, X_{2}$ and $X_{3}$ denote the dimensionless position of the reaction interfaces measured from the center of the pellet, for $\mathrm{Fe}_{3} \mathrm{O}_{4} /$ $\mathrm{Fe}_{2} \mathrm{O}_{3}, \mathrm{FeO} / \mathrm{Fe}_{3} \mathrm{O}_{4}$ and $\mathrm{Fe} / \mathrm{FeO}$, respectively. Thus $X_{1}=X_{2}=X_{3}=1$ denotes zero reaction while $X_{1}=$ $X_{2}=X_{3}=0$ designates the complete reduction of the pellet to sponge iron. The key operating conditions for the computation are given in Table 1.

* Received November 15, 1976.

** Department of Chemical Engineering and Center for Process Metallurgy, State University of New York at Buffalo, Buffalo, N.Y. 14214, U.S.A. On leave from Research Institute of Mineral Dressing and Metallurgy, Tohoku University, Katahira, Sendai 980.

*** Department of Materials Science and Engineering, Massachusetts Institute of Technology, Cambridge, Massachusetts 02139, U.S.A.

$+\quad$ It is likely that the assumption that $u_{s}$ is uniform is an over-simplification. The formulation developed in the previous paper ${ }^{1)}$ allows the consideration of systems, where $u_{s}$ is distributed. An alternative way of allowing for variable solid mass flux, within the computational framework developed here, is to assign an effective spatial distribution to $\varepsilon_{v}$ in Eq. (5). 
Let us now consider the specific cases that were examined.

\section{Case (1) Uniform Mass Velocity of Gas and Isothermal Conditions}

Under these conditions the volumetric gas flow rate, or the linear gas velocity is uniform in radial direction but because the void fraction and the particle diameter are radially distributed, the mass flux of the solids $G_{s}$ will be a function of the axial and the radial coordinates; this in turn will produce spatial variations in gas composition and also in the local extents of reaction. The computed results for this case are shown in Figs. 1 to 3.

Figure 1 shows isopleths of the reaction interfaces and the corresponding values of the mole fraction of hydrogen, throughout the system. It is seen that the isopleths for $X_{1}=0$ and $X_{2}=0$, show little radial distribution; in contrast to the isopleths corresponding to $X_{3}$. This behavior may be readily explained by considering that the equilibrium constants for the reduction steps to magnetite and to wustite are quite high, thus the hydrogen content in the gas much stream is higher than that corresponding to equilibrium conditions. It follows that there is a sufficiently large driving force for these reactions to proceed quite rapidly. In contrast the equilibrium constant for the

Table 1. Numerical values of the process parameters

\begin{tabular}{l|c}
\hline Length of the moving bed & $10 \mathrm{~m}$ \\
Diameter of the bed & $5 \mathrm{~m}$ \\
Apparent density of the pellet & $4120 \mathrm{~kg} / \mathrm{m}^{3}$ (solid) \\
Pressure of top gas & 1 atm \\
Ambient temperature & $20^{\circ} \mathrm{C}$ \\
Inlet solid temperature & $20^{\circ} \mathrm{C}$ \\
Molar fraction of each component of & $0.5\left(X_{\mathrm{H}_{2}}\right)$ \\
inlet gas & $0.5\left(X_{\mathrm{N}_{2}}\right)$
\end{tabular}

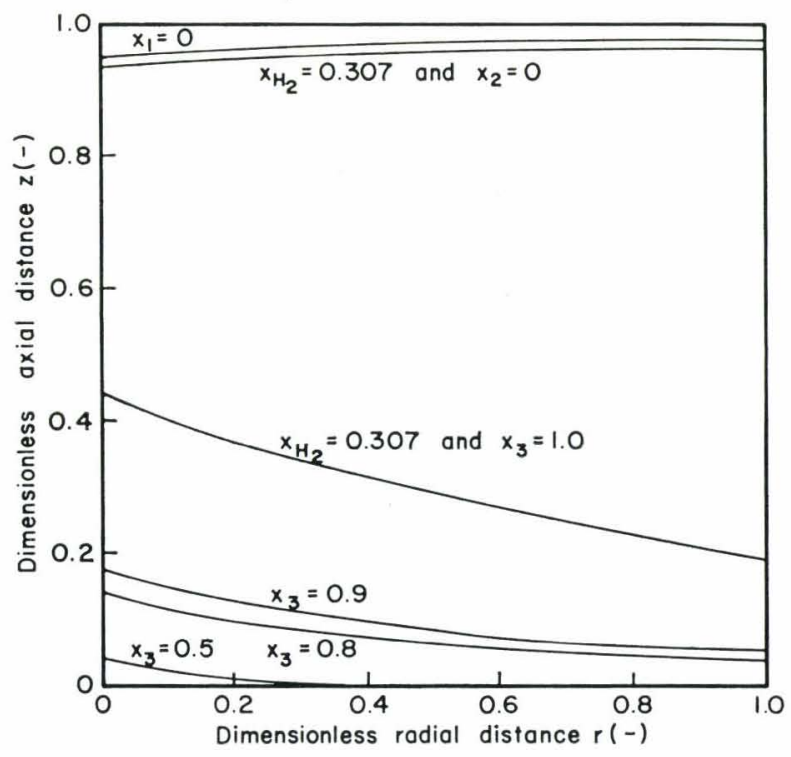

Fig. 1. Contour lines of $X_{\mathrm{H}_{2}}, X_{1}, X_{2}$ and $X_{3}$ for $u_{s}=2.80$, $T_{0}=900, G_{0}=5000$, type (A) for $d_{p}$ and $\varepsilon_{v}$ (uniform gas flow and isothermal conditions) wustite to iron reduction step is much smaller $(0.628$ at $900^{\circ} \mathrm{C}$ ). Because of the solids flow distribution imposed on the system $G_{s z}$ is larger near the walls than in the central core and hence there is a partial hydrogen starvation in the wall region, which retards the wustite to iron reduction step.

Figure 2 shows the axial $X_{\mathrm{H}_{2}}$ profiles for the same system, for three radial positions. The initial drop in the hydrogen content of the gas is due to hydrogen consumed by the hematite to magnetite and magnetite to wustite reduction steps, while the drop in hydrogen levels in the vicinity of $z \approx 1$ is caused by the final reduction step, wustite to iron. The horizontal portion of the curve corresponds to a " chemical reserve zone" where equilibrium is being maintained between the gaseous and the solid streams.

Figure 3 shows the axial distribution of the mass velocity of the solid stream at three radial positions. As expected, Fig. 3 reflects a behavior that corresponds to that seen in Fig. 2. The reduction in the mass velocity of the solids, is of course, caused by the chemical reaction, which has been reflected by the shape of the curves shown in Fig. 2. The difference shown by the ordinates of the horizontal portions of the curves has been imposed on the system, by specifying a distribution in the mass velocity of the solids, by Eq. (5).

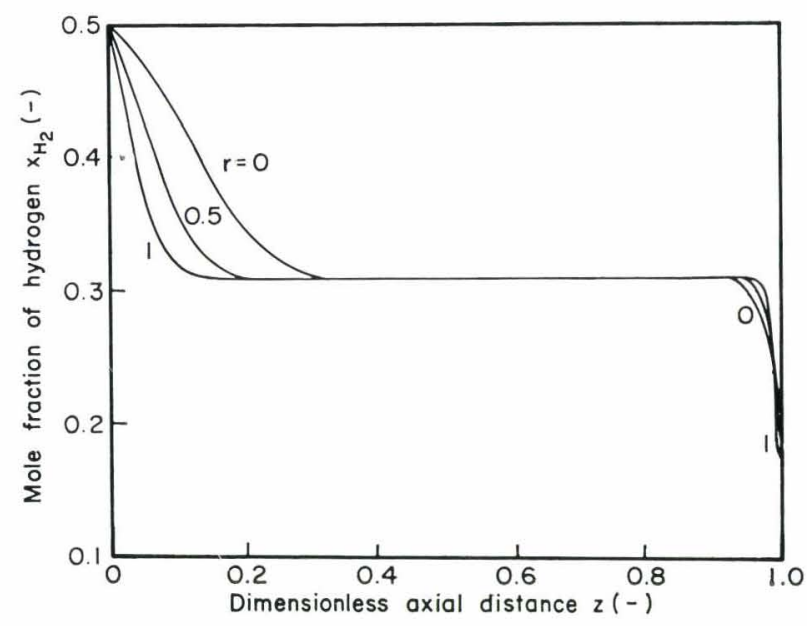

Fig. 2. Axial distributions of $X_{\mathrm{H}_{2}}$ at various $r$ for the same conditions as in Fig. 1

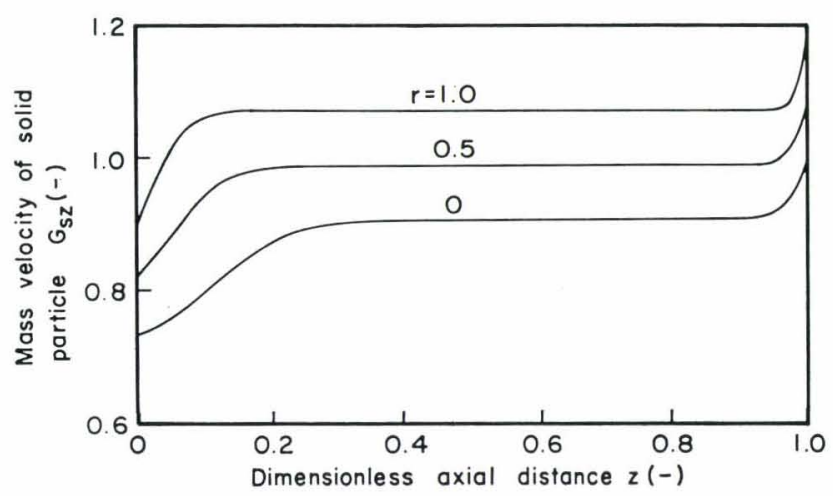

Fig. 3. Axial distributions of $G_{s z}$ at various $r$ for the same conditions as in Fig. 1 


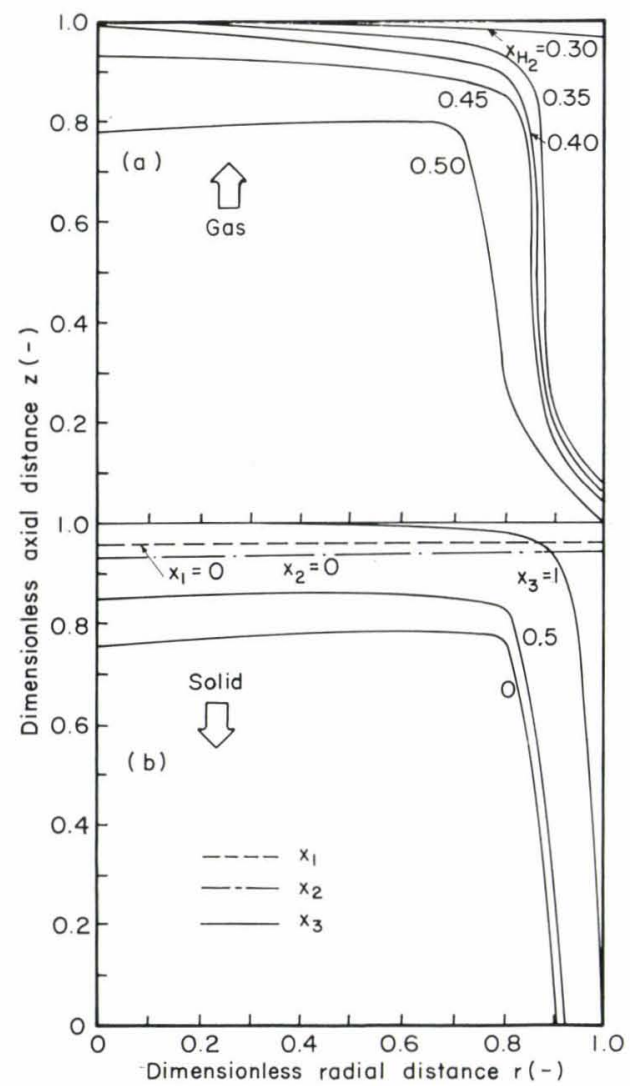

Fig. 4. Contour lines of $X_{\mathrm{H}_{2}}, X_{1}, X_{2}$ and $X_{3}$ for $u_{s}=3, T_{0}=$ $900, G_{0}=9000$, type (A) for $d_{p}$ and $\varepsilon_{v}$ (non-uniform gas flow and isothermal conditions)

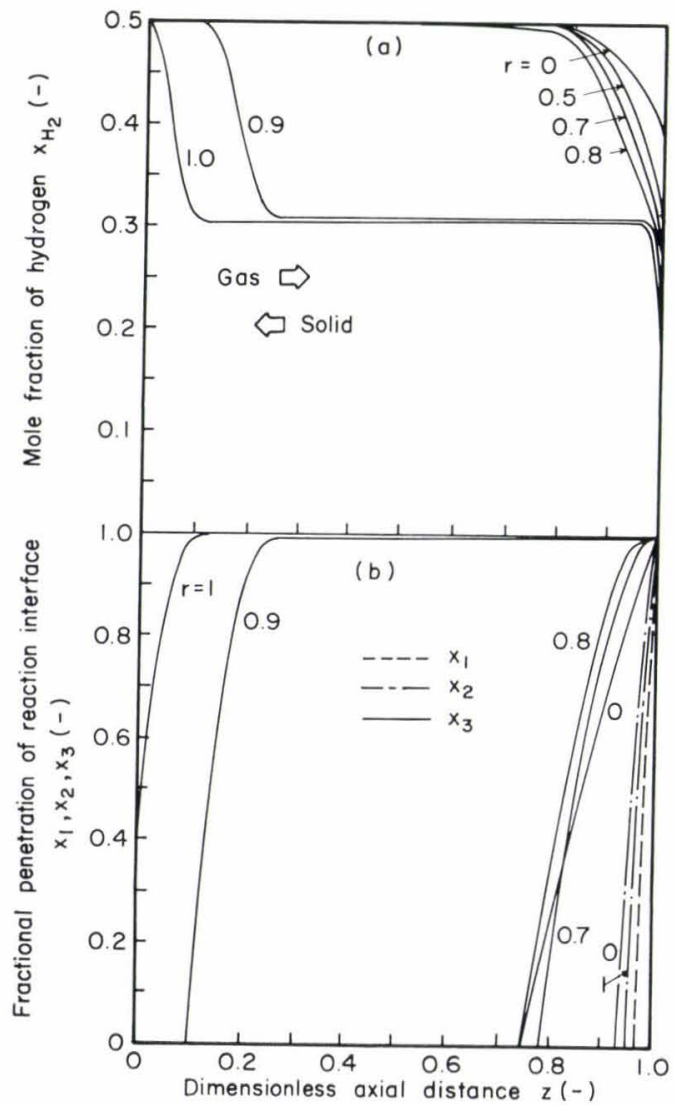

Fig. 5. Axial distributions of $X_{\mathrm{H}_{2}}, X_{1}, X_{2}$ and $X_{3}$ for the same conditions as in Fig. 4 investigators, ${ }^{2,3)}$ who studied counter-current moving responds to the predictions made by many previous

The idealized behavior, shown in Figs. 1 to 3 corbed reaction systems by using one-dimensional mathematical model, therefore to examine the more realistic, but more complex cases, in the following.

\section{Case (2) Non-uniform Gas Flow and Isothermal Con- ditions}

The behavior of these systems is illustrated in Figs, 4 to 7,9 to 12 , and 14 to 18 , while Figs. 8 and 13 depict these identical systems, but for uniform gas flow.

Figures 4 to 7 describe a system where the void fraction of the bed decreases radially from the center toward the wall region, thus giving preferential flow of the gases in the central core, and an increase mass velocity of the solid stream near the walls. The consequences of this spatial mismatch of the two streams are illustrated quite dramatically in Fig. 4, showing the isopleths for the mole fraction of hydrogen and the three reaction fronts. The very marked nonuniformity of these is readily apparent. Hydrogen is rapidly consumed in the wall region and as a result the progress of the reduction process to iron is appreciably retarded here.
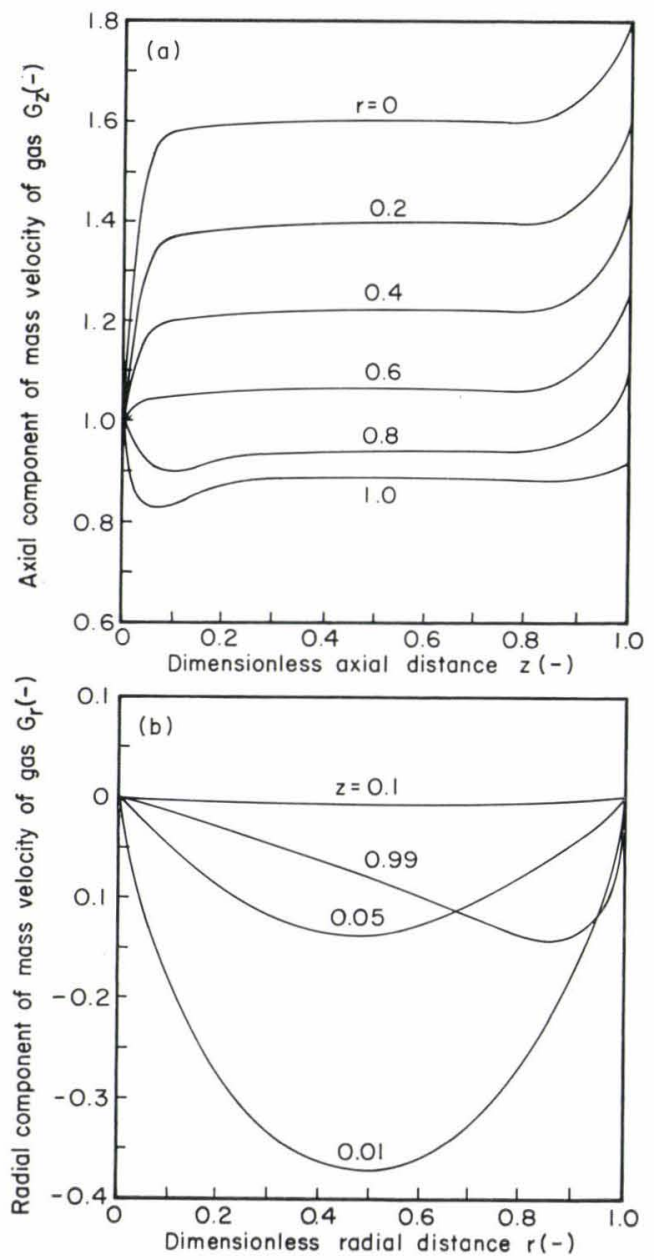

Fig. 6. Axial and radial distributions for respective component of mass velocity of gas for the same conditions as in Fig. 4 
Figure 5 shows the axial profiles of $X_{\mathrm{H}_{2}}$ and of the reaction fronts for various values of the dimensionless radial distance from the central core. The very marked effect of the radial position on these isopleths is again readily apparent.

Figure 6 shows the axial and radial profiles of the mass velocity of the gas. Figure 6 (a) indicates sharp changes in the value of $G_{z}$ at the inlet and at the exit of the bed; in general these correspond to the consumption of the hydrogen by the three step chemical reaction. However, there is another complicating factor, the effect of which is apparent on the left hand side of Fig. 6 (a) and also on Fig. 6 (b). For the case considered, the structure of the bed (high resistance near the wall, lower resistance to flow in the central portion) causes gas flow from the wall region to the central portion of the bed, in the vicinity of the gas inlet. The uneven rate at which the particles react in the system, produces a similar effect. Figures 7 (a) and (b) show the axial mass velocity of the solid stream, with the axial position and the radial position as parameters. The radial variations in the $G_{s z}$ are caused by both the prescribed profiles of the void fraction and by the progress of the reaction, which, of course changes the density of the solid particles. We note in Fig. 7 (b) that the values of $G_{s z}$ near the wall $(r=1.0$ and 0.9$)$ are markedly different from those in the rest of the bed.
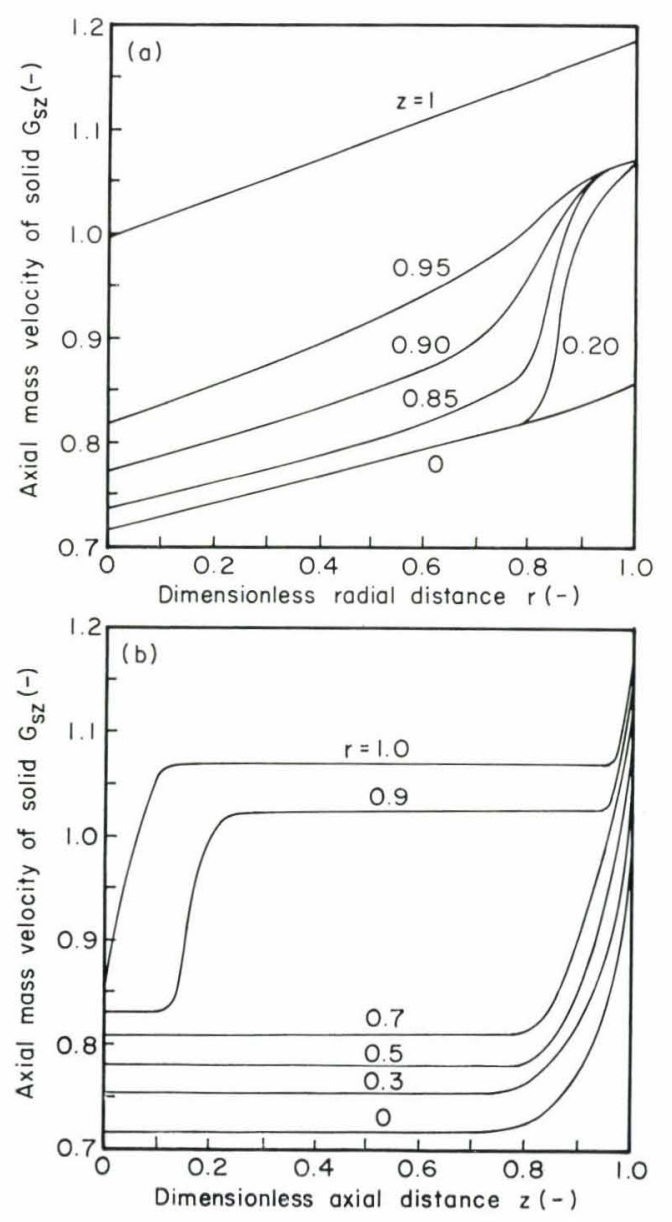

Fig. 7. Axial and radial distributions for mass velocity of solid for the same conditions as in Fig. 4

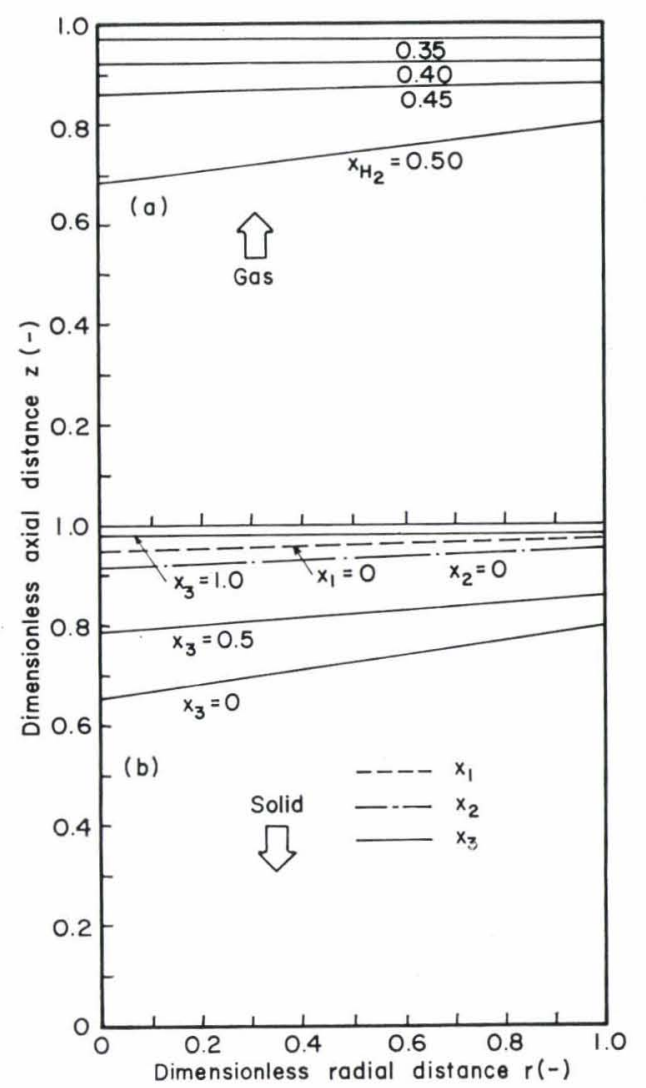

Fig. 8. Contour lines of $X_{\mathrm{H}_{2}}, X_{1}, X_{2}$ and $X_{3}$ for the same conditions as in Fig. 4 (uniform gas flow and isothermal conditions)

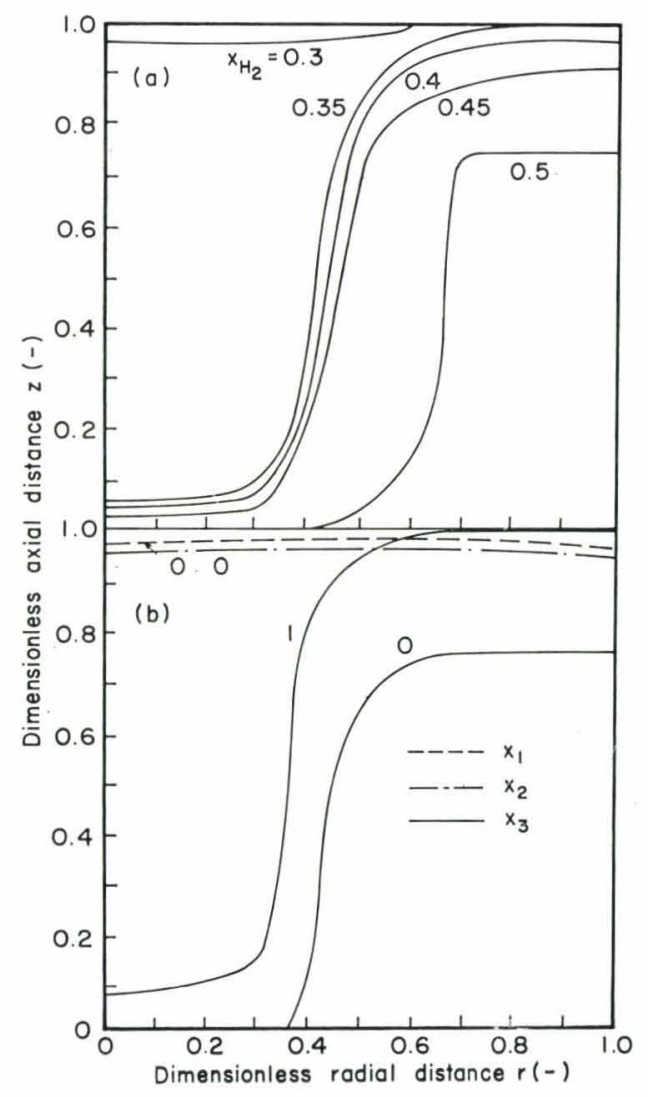

Fig. 9. Contour lines of $X_{\mathrm{H}_{2}}, X_{1}, X_{2}$ and $X_{3}$ for $u_{s}=3, T_{0}=$ $900, G_{0}=9000$, type (B) for $d_{p}$ and $\varepsilon_{v}$ (non-uniform and isothermal conditions) 


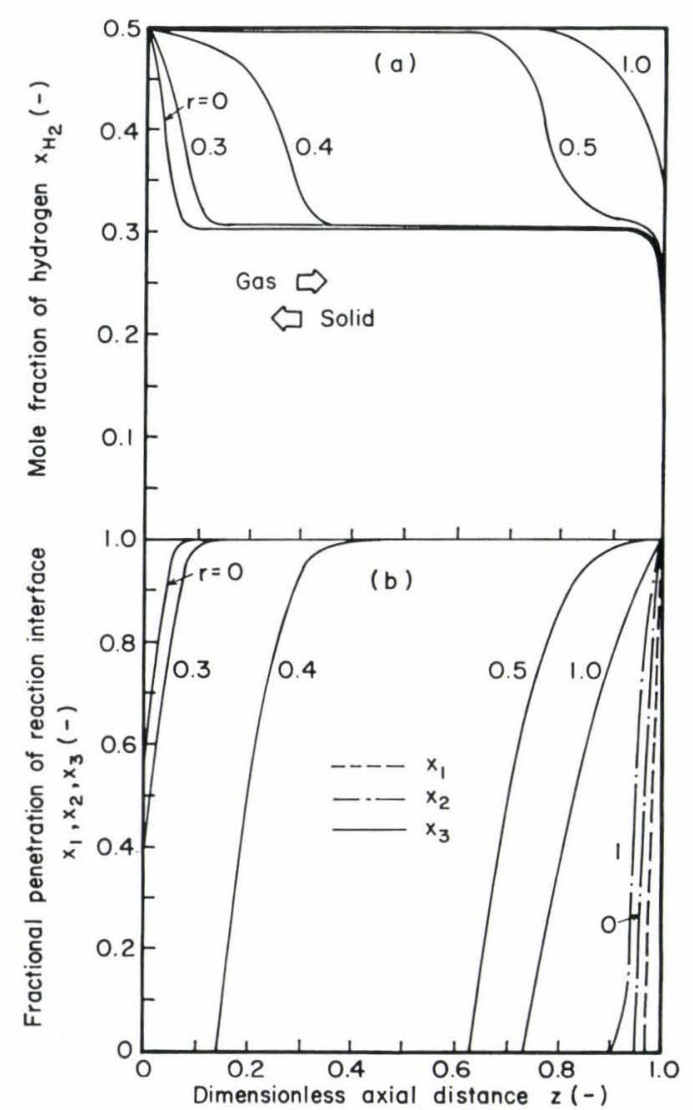

Fig. 10. Axial distribution of $X_{\mathrm{H}_{2}}, X_{1}, X_{2}$ and $X_{3}$ for the same conditions as in Fig. 9
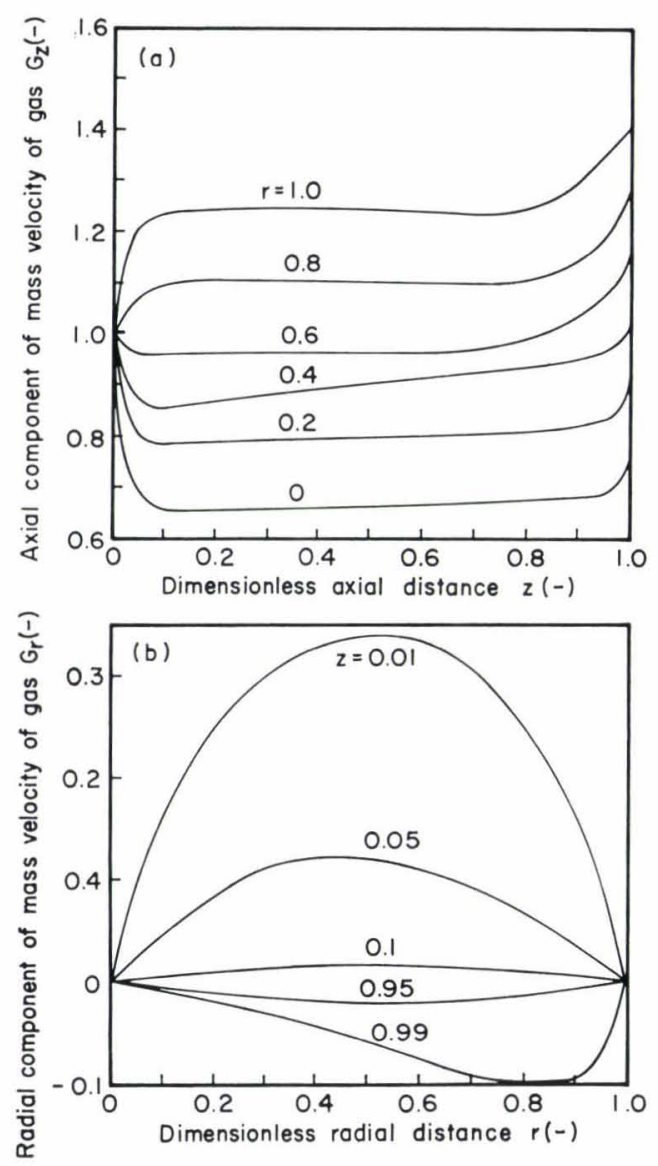

Fig. 11. Axial and radial distributions of respective component of mass velocity of gas for the same conditions as in Fig. 9
It is of interest to contrast the plots shown in Figs. 4 to 7 with the profiles given in Fig. 8 computed for uniform flow, for otherwise identical conditions. It is obvious that the assumption of uniform flow would very seriously misrepresent the behavior of this system.

Figures 9 to 12 show a set of computed results, for a void fraction and particle size distribution, as given by Eqs. (3) and (4). Under these conditions there exists a preferential gas flow in the vicinity of the walls, while the mass velocity of the solids is higher in the central core. This mismatch of the gaseous and the solid streams, which is probably a closer approximation to physical reality than the previously discussed case, results in a very marked distortion in the concentration profiles. Figure 9 (a) indicates the preferential depletion of hydrogen in the central core, while as seen in Fig. 9 (b), the reduction of wustite to iron (the most sensitive component of the reduction sequence) proceeds faster in the wall region than in the central core.

The axial concentration profiles, given in Fig. 10 indicates incomplete reduction in the central core. Figure 11, a plot of the axial and radial components of the mass velocity of the gas, depicts trends that are essentially the opposite of what was seen in Fig. 6 for preferential gas flow in the central core.

In Fig. 11, there is a radial flow of the gas from the central core to the wall region at the bottom of the
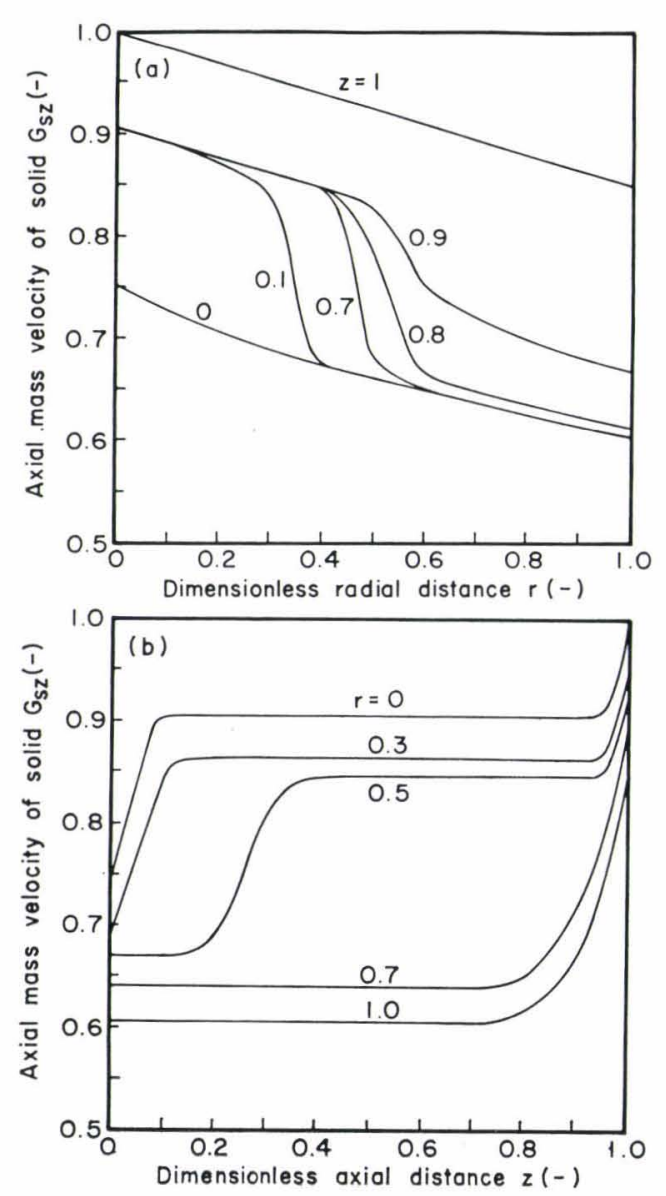

Fig. 12. Axial and radial distriutions of mass velocity for the same conditions as in Fig. 9 


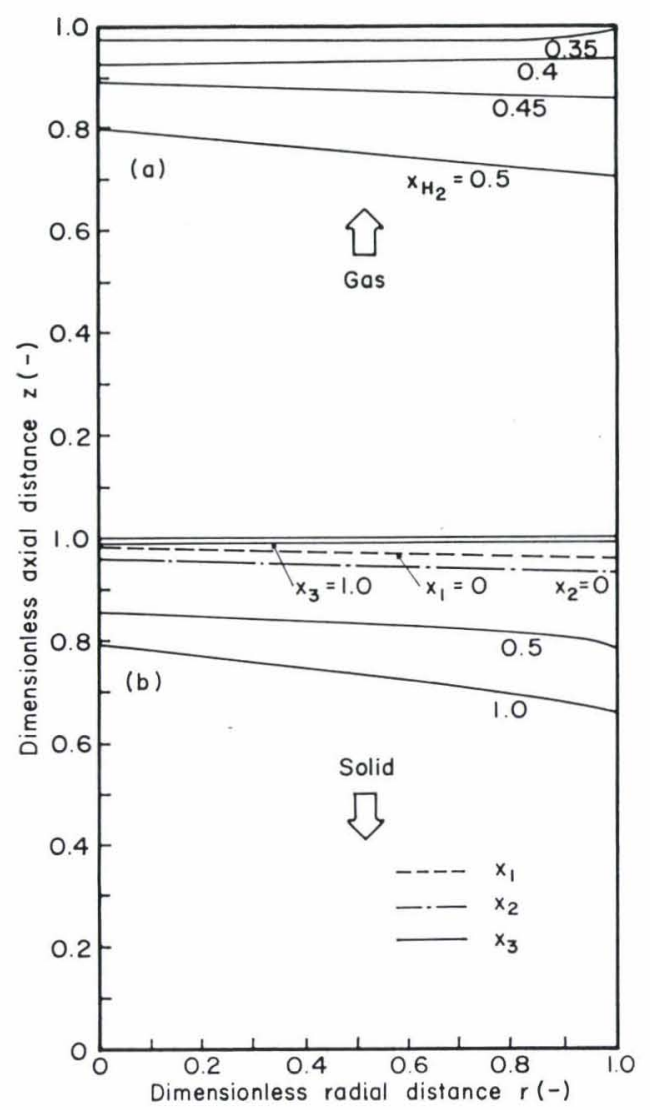

Fig. 13. Contour lines of $X_{\mathrm{H}_{2}}, X_{1}, X_{2}$ and $X_{3}$ for the same conditions as in Fig. 9 (uniform and isothermal conditions)

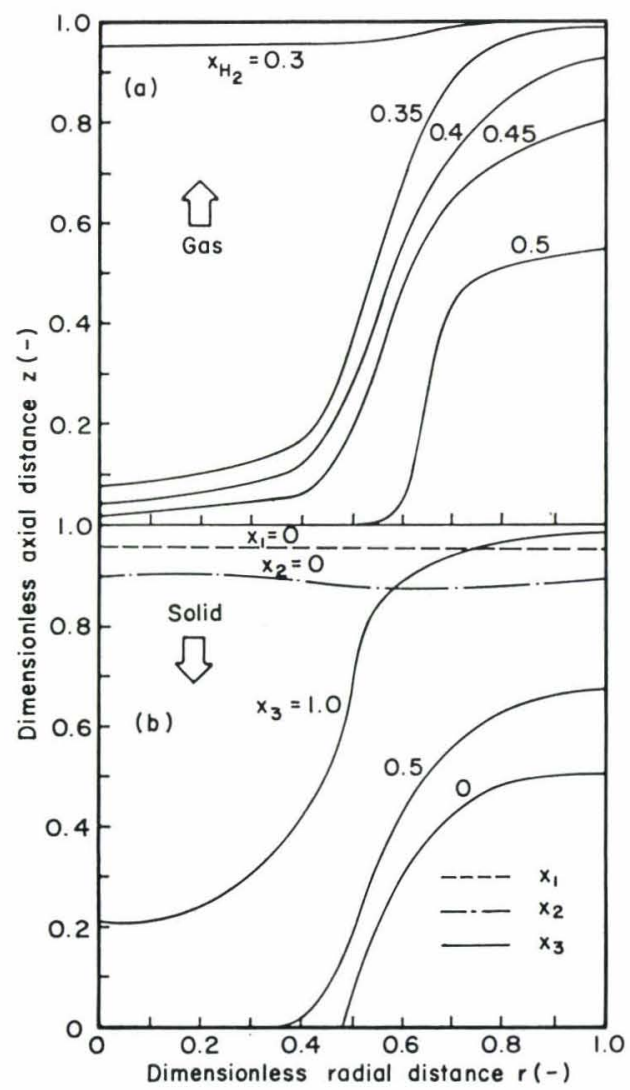

Fig. 14. Contour lines of $X_{\mathrm{H}_{2}}, X_{1}, X_{2}$ and $X_{3}$ for $u_{s}=3, T_{0}$ $=800, G_{0}=9000$, type (B) for $d_{p}$ and $\varepsilon_{v}$ (non-uniform and isothermal conditions) bed, as caused by the bed resistance, while the trend is reversed at the top of the bed, as a result of the hydrogen consumption, due to the chemical reactions.

Figure 12, depicting the mass velocity of the solid stream indicates that the hematite is reduced to metallic iron at about $z=0.8$ in the wall region, while the reaction is not completed even at the exit in the central core.

Figure 13 depicts profiles, computed for uniform gas flow, but otherwise identical conditions to those used in the computation of Figs. 9 to 12. It is readily seen that the assumption of uniform gas flow would seriously misrepresent the behavior of the system.

Figures 14 and 15 show a set of computed results for the same operating conditions as those given, previously in Figs. 9 to 12, but for operation at $800^{\circ} \mathrm{C}$. In contrast Figs. 16 and 17 depict the behavior of the same system, but at $1000^{\circ} \mathrm{C}$. These three sets of figures are qualitatively similar; however the effect of gas maldistribution, as caused by the difference in the rates of the chemical reactions, is the most severe at $1000^{\circ} \mathrm{C}$, and is the least severe at $800^{\circ} \mathrm{C}$; this is to be expected because the rates of the reactions are appreciably faster at $1000^{\circ} \mathrm{C}$ than at $800^{\circ} \mathrm{C}$. It is to be stressed here, that gas flow maldistribution is caused by the spatially distributed chemical reaction rates which manifests itself particularly at
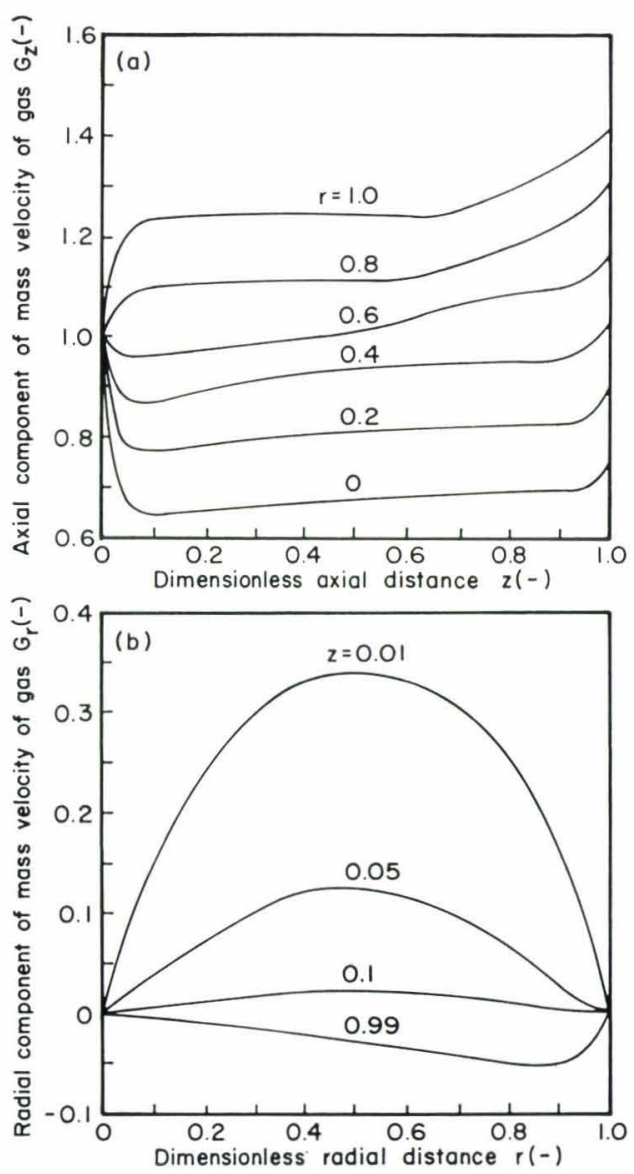

Fig. 15. Axial and radial distributions of the respective component of mass velocity of gas for the same conditions as in Fig. 14 


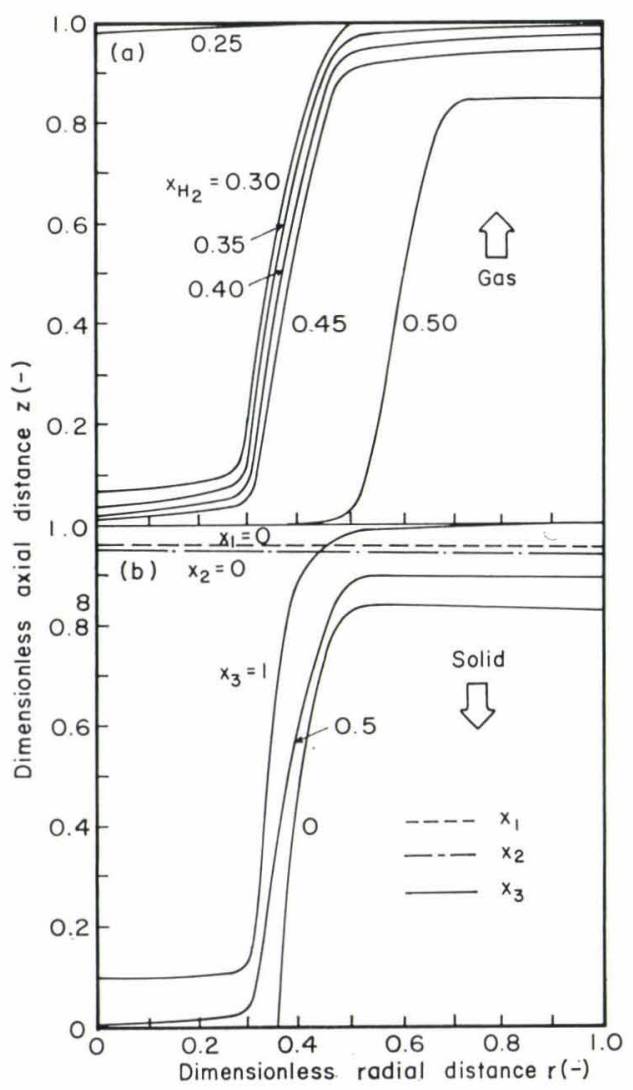

Fig. 16. Contour lines of $X_{\mathrm{H}_{2}}, X_{1}, X_{2}$ and $X_{3}$ for $u_{s}=3, T_{0}$ $=1000, G_{0}=9000$, type (B) for $d_{p}$ and $\varepsilon_{v}$ (nonuniform and isothermal conditions)

the top of the bed, as seen in Figs. 11 (b), 15 (b) and 17 (b).

Figure 18 shows a plot of the pressure profiles, computed for Cases (A) and (B), as indicated in the captions. The appreciable difference seen between these two systems is readily explained by the fact that for the cylindrical coordinates used the surface area corresponding to the wall region receives a much greater weighting than the central core. It follows that Case (A) where the resistance to flow is the highest in the wall region will result in a higher overall pressure drop; however, this is just an artifact of the functional relationship assigned to the spatial distribution of $\varepsilon_{v}$ and $d_{p}$.

\section{Case (3) Distributed Gas Flow and Non-isothermal Behavior}

It has been seen in discussion of the previous cases that the distributed nature of the gas flow can have a very marked effect in causing a significant departure from the radial concentration profiles. In many real, industrial reactors the situation is further complicated by the fact that the system is non-isothermal.

In the following we shall examine the behavior of non-isothermal system; however, in order to reduce the computer time requirements to manageable proportions, the heat flow and the fluid flow equations will be uncoupled; more specifically, we shall assume that the axial component of the mass velocity of the gas is given by:
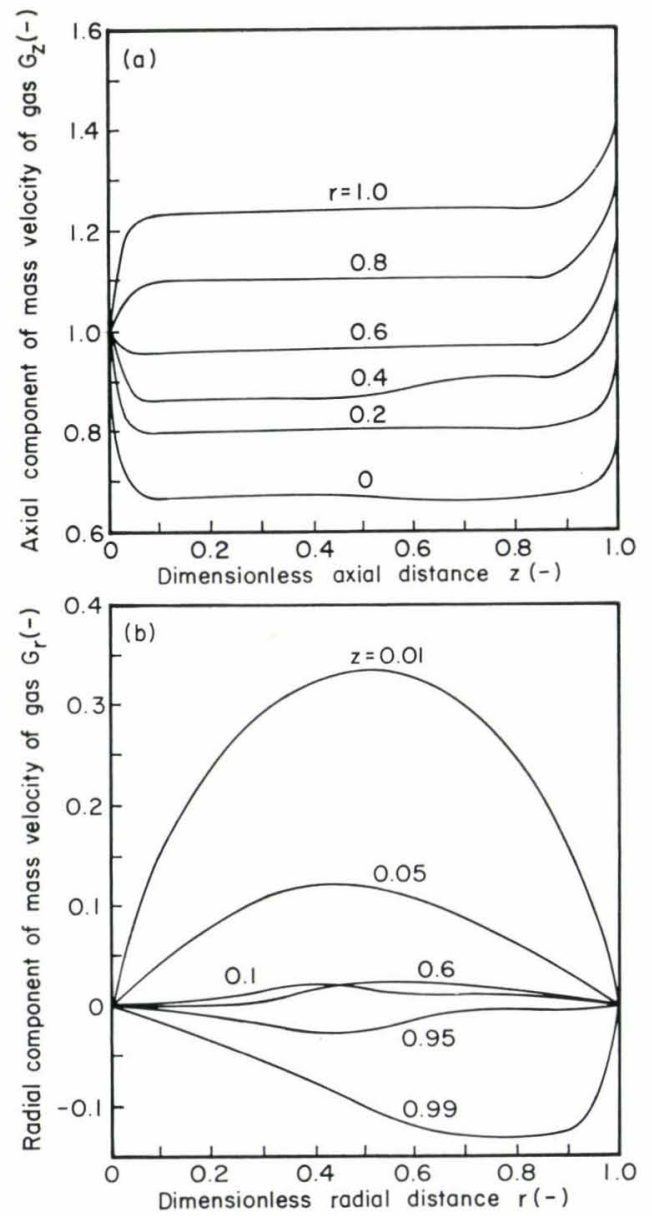

Fig. 17. Axial and radial distributions of the respective components of mass velocity of gas for the same conditions as in Fig. 16

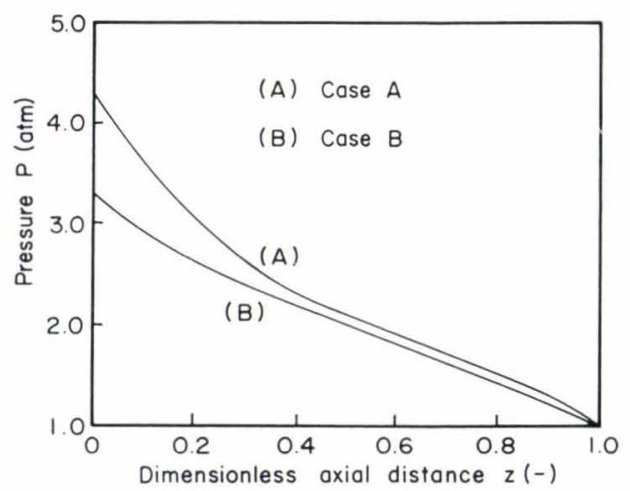

Fig. 18. Axial pressure distributions for $u_{s}=3, T_{0}=900, G_{0}$ $=9000$, and (1); type (A), (2): type (B) for $d_{p}$ and $\varepsilon_{v}$ (non-uniform and isothermal conditions)

$$
G=\left(\varepsilon_{v} / \varepsilon_{v 0}\right) G_{z 0}
$$

where the subscript 0 referes to conditions in the axis of the bed.

A selection of the computed results is shown in Figs. 19 to 24 . It is of interest to compare the plots shown in Fig. 19, with the corresponding graphs, given earlier, for isothermal conditions. It is seen that the temperature distributions computed from the heat balance equations do modify the reduction profiles; in particular the reaction zones for the hem- 


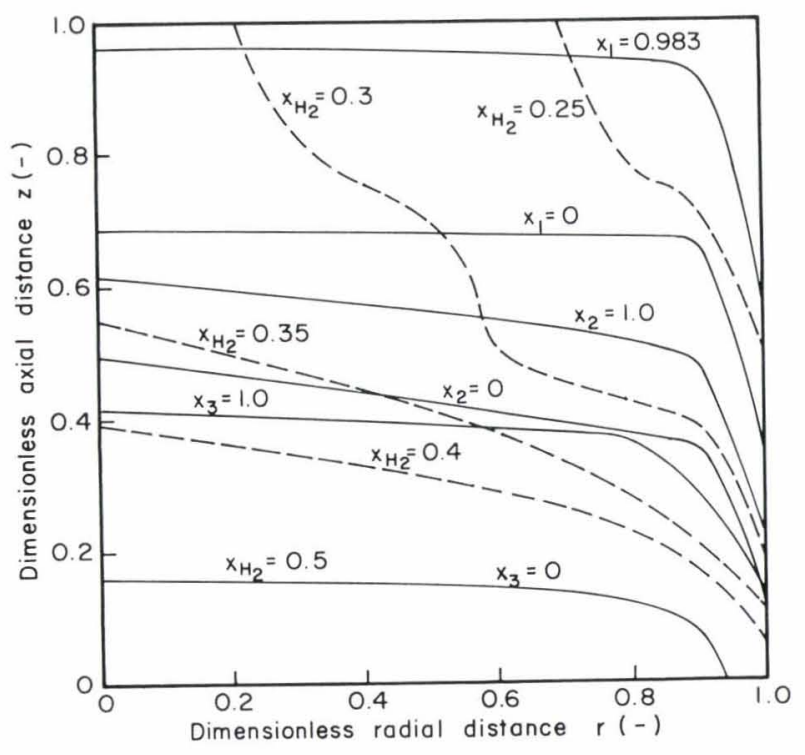

Fig. 19. Contour lines of $X_{\mathrm{H}_{2}}, X_{1}, X_{2}$ and $X_{3}$ for $u_{s}=2, T_{0}$ $=1000, G_{0}=5640$, type (A) for $d_{p}$ and $\varepsilon_{v}$ (distributed gas flow and non-isothermal conditions)

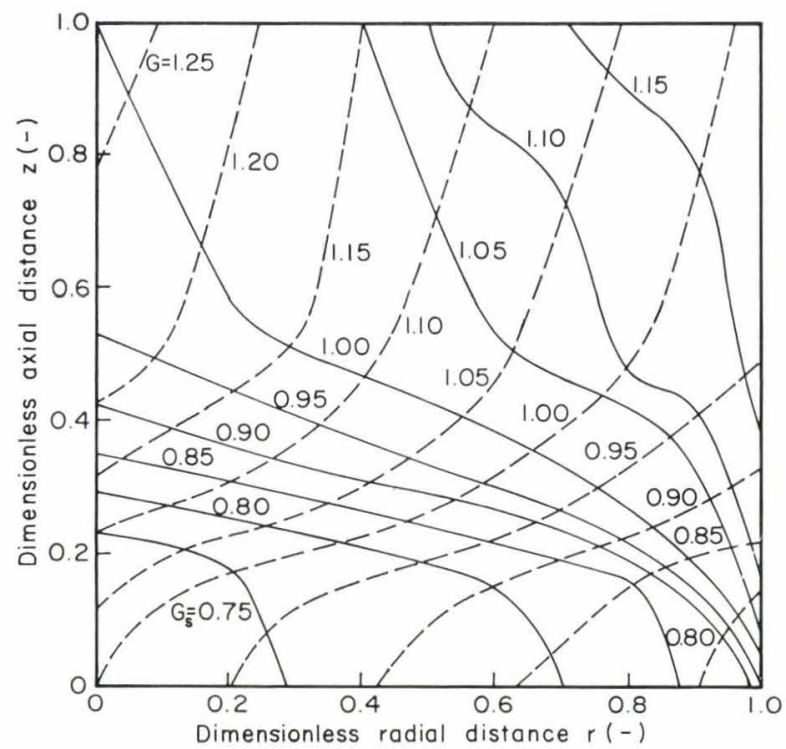

Fig. 20. Contour lines of $G$ and $G_{s}$ for the same conditions as in Fig. 19

atite to magnetite step and for the magnetite to wustite step tend to be wider than given by the computed values for the isothermal case.

Figure 20 indicates very marked non-uniformities in the profiles of both the gas and the solid mass velocities. The radial temperature distributions in the gaseous and in the solid streams are given in Fig. 21, where it is seen that the solid and the gas temperatures are quite close, moreover there exist quite sharp temperature gradients in the vicinity of the walls.

In Fig. 22 we present computed axial profiles of $X_{\mathrm{H}_{2}}$ and of the mass velocity of the solids, with the dimensionless radial coordinate as a parameter. The marked radial gradients seen in this graph are at least in part attributable to the radial temperature gradients, caused by heat losses through the wall.

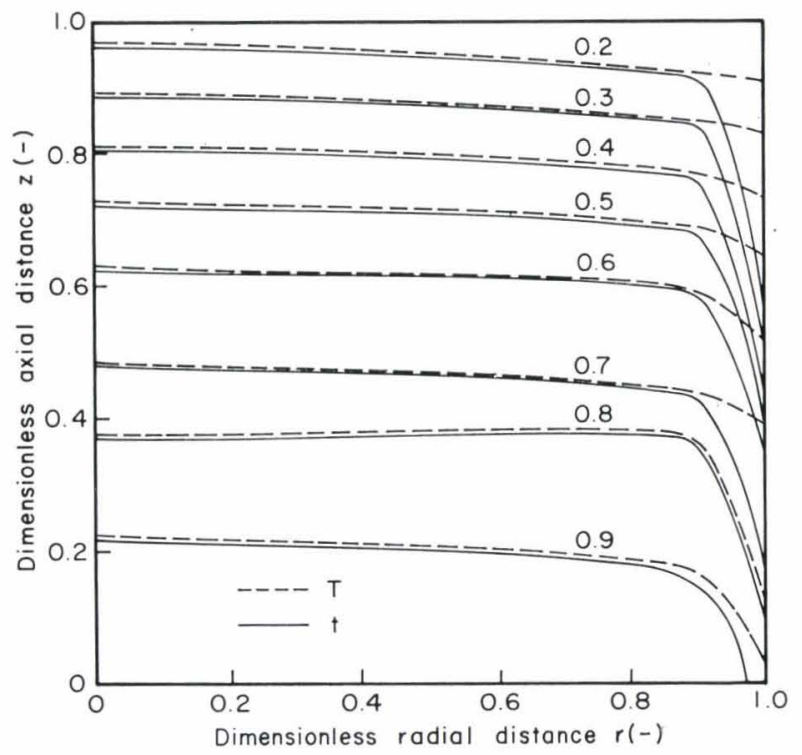

Fig. 21. Isothermal lines of gas and solid for the same conditions as in Fig. 19
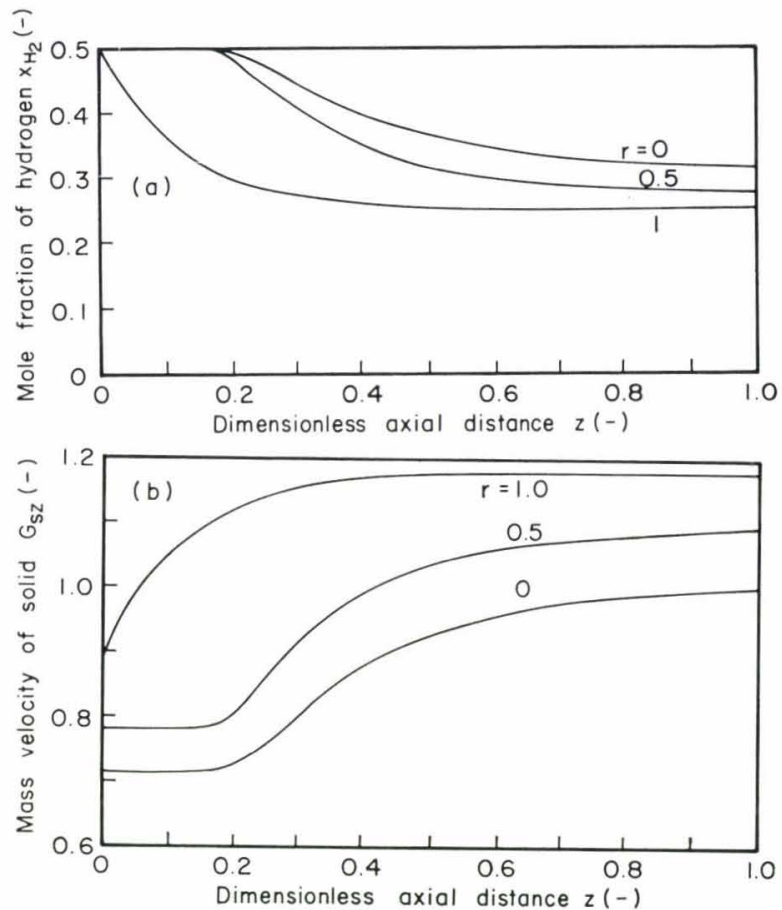

Fig. 22. Axial distributions of $X_{\mathrm{H}_{2}}$ and $G_{s z}$ for the same conditions as in Fig. 19

The axial profiles of the three reaction interfaces, given in Fig. 23 indicate that the reactions proceed in different locations from those found for the isothermal systems, particularly, because of the cooler regions in the vicinity of the walls. It is instructive to examine the axial profiles of the (dimensionless) gas temperature; the breaks in the curves are attributable to the effect of the endothermic heat of reaction.

It may be more appropriate to approximate the distribution of the mass velocity of the gas, by the following expression:

$$
G_{z}=\left(\sqrt{\left.\varepsilon_{v}^{3}\left(1-\varepsilon_{v 0}\right) d_{p} / \varepsilon_{v 0}^{3}\left(1-\varepsilon_{v}\right) d_{p 0}\right)} \cdot G_{z 0}\right.
$$




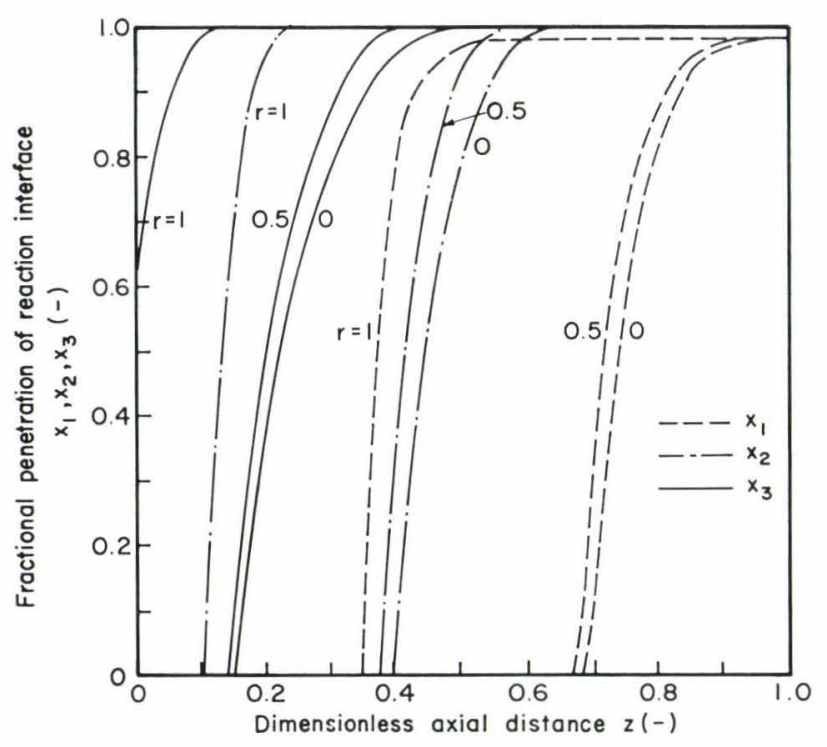

Fig. 23. Axial distributions of $X_{1}, X_{2}$ and $X_{3}$ for the same conditions as in Fig. 19

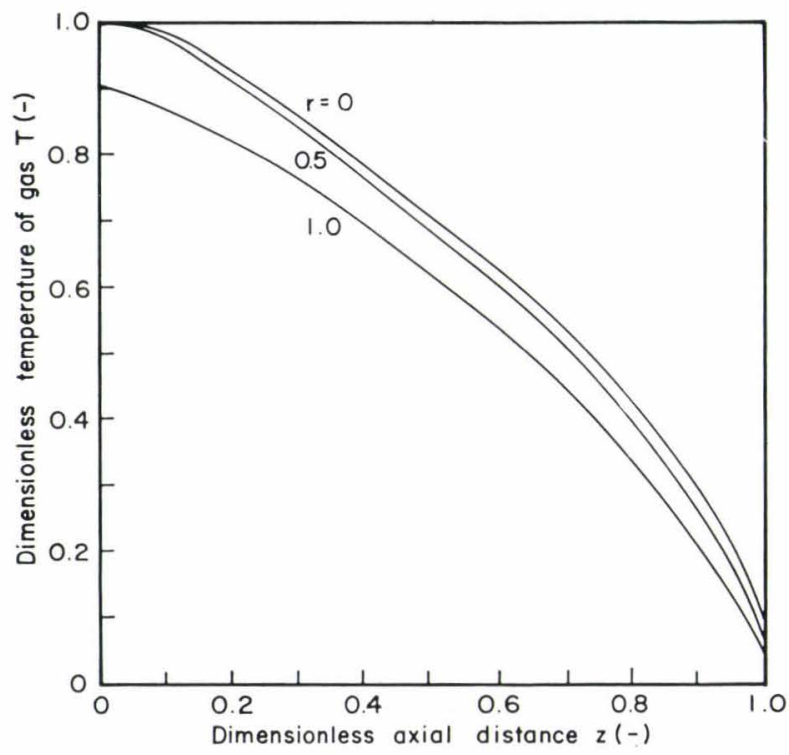

Fig. 24. Axial distribution for the temperature of gas for the same conditions as in Fig. 10

which is based upon the postulate that the ratio of the velocities is inversely proportional to the flow resistance. Results were also computed on this basis and they show qualitatively similar trends to those exhibited by Figs. 19 to 24; for this reason these are not reproduced here.

\section{Concluding Remarks}

In the previous paper, a general formulation has been presented, describing the behavior of a system in which iron oxide pellets are reduced by hydrogen in a counterflow arrangement, such that both the gas and the solids streams may be maldistributed. In the present paper a comprehensive set of computed results are presented depicting three basic situations, namely:

1) Uniform gas flow and isothermal conditions

2) Non-uniform gas flow and isothermal condi- tions

3) Distributed gas flow and non-isothermal conditions.

System (1) corresponds to those computed on the assumptions usually made in the modeling of gassolid reactions in moving beds, thus it represents the basis for comparison.

The maldistribution in the gas and the solids flows was generated by assigning a spatially variable void fraction and solid particle diameters to these systems. Clearly a high void fraction near the walls resulted in a preferential gas flow near the wall region, while the corresponding mass velocity of the solids was reduced in the same zone. The reverse is also true; a low void fraction in the wall region caused preferential gas flow in the central core and preferential solids flow (i.e., mass velocity) in the wall regions.

The spatial variations in particle diameter, assigned to the system caused both variable resistances to gas flow and non-uniformities in the local rates of reaction, due to the obvious variations in the interfacial areas.

The systematic examination of the effect of these process variables revealed that gas maldistribution can lead to a very marked maldistribution of the concentration isopleths and of the local extents of reaction. This situation can become particularly serious, when the gas and the solids streams are mismatched, i.e., through preferential flow of the gas in the wall regions, and preferential solids flow in the central core, which is of likely occurrence in many industrial systems. Under these conditions the frequently made assumption of uniform gas and solids flows would very markedly misrepresent the system and the reliance on these assumptions could cause serious design errors.

The examination of non-isothermal behavior has shown that axial temperature gradients may have a marked effect on the overall performance of the system, but that overall radial gradients have a much less important modifying effect.

In conclusion it may be stated that through the presentation of a comprehensive set of computed data it was demonstrated that gas and solid flow maldistribution plays a very important role in affecting the performance of moving bed reactors, particularly, when a mismatching of the gas and the solids streams occurs. The examples computed in the present paper were concerned with the reduction of iron oxide pellets with hydrogen; it is felt, however, that similar considerations may be applied to a broad range of direct reduction systems, and also to the stack region of the blast furnace. It is thought that flow maldistribution phenomena are of great practical importance and their further study would be fully justified.

\section{Nomenclature}

$d_{p}, d_{p 0}$ : Particle diameter and it at central axis in the reactor $(\mathrm{m})$

$G_{z}, G_{z 0}$ : Axial mass velocity of gas and it at central axis

$G_{r}: \quad$ Radial mass velocity of gas 
$G_{s z}$ : Axial mass velocity of solid particles

$G_{s 0}^{\prime}$ : Mass velocity of solid particles at the top of the reactor $\left(\mathrm{kg} / \mathrm{m}^{2} \cdot \mathrm{hr}\right)$

$G_{0}$ : Average mass velocity of inlet gas $(\mathrm{kg} /$ $\mathrm{m}^{2} \cdot \mathrm{hr}$ )

$P$ : Pressure of gas $(\operatorname{atm}(\mathrm{a}))$

$r$ : Radial distance from the central axis

$T$ : Temperature of gas

$T_{0}$ : Temperature of inlet gas $\left({ }^{\circ} \mathrm{C}\right)$

$t$ : Temperature of solid particles

$u_{s}$ : Linear velocity of solid particle $(\mathrm{m} / \mathrm{hr})$

$X_{\mathrm{H}_{2}}$ : Mole fraction of hydrogen

$X_{1}, X_{2}, X_{3}$ : Dimensionless position of the reaction interfaces between hematite and magnetite, magnetite and wustite, and wustite and iron $\varepsilon_{v}, \varepsilon_{v 0}$ : Voidage of the bed and it at the central axis

$\rho_{a}$ : Apparent density of the pellet $\left(\mathrm{kg} / \mathrm{m}^{3}\right.$ (solid))

\section{Acknowledgements}

The authors wish to thank the A.E. Anderson Foundation for partial support of this investigation.

\section{REFERENCES}

1) J. Yagi and J. Szekely: Trans. ISIJ., 17 (1977), 569.

2) J. Yagi, A. Moriyama and I. Muchi: J. Japan Inst. Metals, 32 (1967), 209.

$3)$ R. H. Spitzer, F. S. Manning and W. O. Philbrook: Trans. Met. Soc., AIME, 242 (1968), 618. 